The Astrophysical Journal, 175:819-829, 1972 August 1

(C) 1972. The American Astronomical Society. All rights reserved. Printed in U.S.A.

\title{
PHOTOSPHERIC FACULAE AND THE SOLAR OBLATENESS
}

\author{
Gary A. Chapman \\ San Fernando Observatory, Space Physics Laboratory, The Aerospace Corporation
}

AND

ANDREW P. INGERSOLL

Division of Geological and Planetary Sciences, California Institute of Technology, and Hale

Observatories, Carnegie Institution of Washington, California Institute of Technology

Received 1971 September 13; revised 1972 February 1

\begin{abstract}
Photospheric faculae near the equatorial solar limb may provide the excess brightness which Ingersoll and Spiegel showed would explain Dicke and Goldenberg's oblateness measurement. Three lines of evidence support this statement: (1) the excess emission of faculae may arise in optically thin regions, as required by the Ingersoll-Spiegel hypothesis; (2) faculae are sufficiently widespread on the solar surface to account quantitatively for the observed signal; and (3) temporal fluctuations in the expected signal due to faculae in 1966 are correlated with fluctuations in the observed signal at the 1 percent level. (The probability of the correlation coefficient for uncorrelated data exceeding the observed value is less than 1 percent.) Although this evidence clearly demonstrates that faculae make a sizable contribution to the observed oblateness signal, it does not preclude an equally sizable contribution due to true gravitational oblateness. Evidence that faculae may not be the only source of oblateness signal comes from the apparent fact that the ratio of fluctuation amplitude to mean signal amplitude is greater for the facular signal than for the observed oblateness signal. However, this difference may be due to errors in reading the photographs from which the facular signal was derived, or to differences in processing the two sets of data. A better test of our hypothesis cannot be made until the daily oblateness signals and their standard deviations are available. In any case, it appears that further data analysis will be necessary before a reliable value of the solar oblateness can be inferred.
\end{abstract}

\section{INTRODUCTION}

By measuring the shape of the solar disk in visible light, Dicke and Goldenberg (1967) inferred a value of the solar oblateness which is 5 times the value expected under the assumption that the entire Sun is rotating with the angular velocity of the seen layers. From this they concluded that the excess perihelion motion of Mercury is not in accord with Einstein's prediction based on general relativity. However, it is possible that their observations do not indicate a true gravitational oblateness, but are simply the result of an excess brightness at the equatorial solar limb. Such brightness might be due to the presence of faculae and sunspots, although Dicke $(1970 a, b)$ concluded that their effect on the oblateness determination is insignificant. ${ }^{1}$ In this paper, we reexamine Dicke's argument, and conclude that his reasons for rejecting faculae as a source of oblateness signal are unjustified. We also find that faculae account for most of the qualitative and quantitative features of Dicke and Goldenberg's 1966 observations. We feel that further observations may be necessary before a reliable value of the solar oblateness can be inferred.

The oblateness observations were made by projecting an image of the Sun on a circular occulting disk and measuring the light flux from the part of the Sun exposed beyond the disk. The part of the flux which varied from equator to pole is the "signal" used to infer the solar oblateness. To distinguish between the effects of brightness

${ }^{1}$ Dr. Dicke informed us (Dicke 1971a) that he had carried out statistical analyses of the effects of faculae and sunspots on the oblateness signal. 
variation and true oblateness, Dicke and Goldenberg observed with three different values of $\delta$, the radial angular distance from the edge of the occulting disk to the mean solar limb. Were the signal the result of a real difference between the equatorial and polar radii of the Sun, the signal amplitude would be substantially independent of $\delta$. Were the signal the result of brightness variation, the amplitude would depend on the amount of exposed area, and hence on $\delta$. Dicke and Goldenberg assumed that any contribution to the signal from brightness variation would be proportional to $\delta$, which dependence, they state, is not consistent with their data. However, Ingersoll and Spiegel (1971) showed that brightness variation in an optically thin region above the top of the photosphere could lead to signal amplitude proportional to $\delta^{1 / 2}$, and such dependence appears to be consistent with the data. This dependence arises because, for the small values of $\delta$ used in the experiments, the area of the solar surface in the field of view from the occulting disk to the limb is proportional to $\delta^{1 / 2}$. And for optically thin regions, the contribution to the signal is simply proportional to the number of emitters in the field of view, which is proportional to solar surface area and therefore to $\delta^{1 / 2}$.

\section{DEPENDENCE ON $\delta$}

Our hypothesis is that the excess oblateness signal measured by Dicke and Goldenberg is due to the presence of faculae near the equatorial solar limb. We first show that faculae could give rise to a signal with amplitude proportional to $\delta^{1 / 2}$. As explained above, such dependence requires that the excess intensity of a facula originate in an optically thin region above the top of the photosphere. We shall present an idealized facular model which meets these conditions; we do not claim that ours is the only model consistent with observation, or that all tenable models lead to a $\delta^{1 / 2}$-dependence.

Our facular model is chosen to fit observations of facular contrast $\Delta I / I$ for faculae at various distances from the solar limb. In continuum light, faculae appear as bright patches of approximately $750 \mathrm{~km}$ diameter. Figure 1 presents observations of $\Delta I / I$ based on the maximum intensity of a facula relative to the surrounding photosphere. The abscissa is $\mu=\cos \theta$, where $\theta$ is the angle between the solar radius vector and the line of sight. The observations are from Rogerson (1961) and Chapman (1970). Our

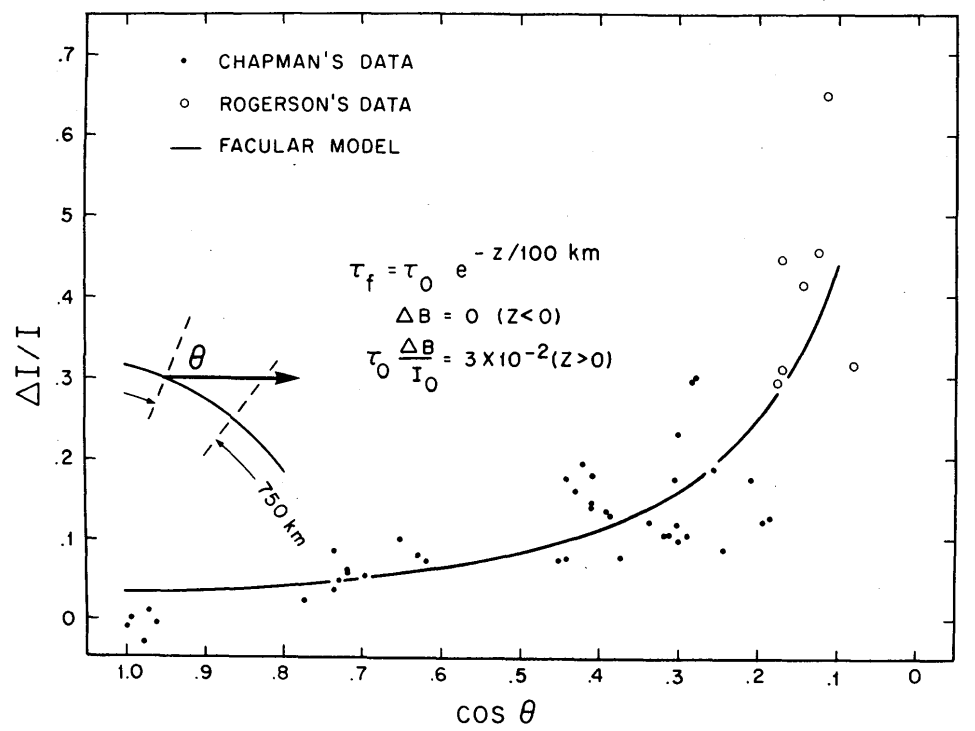

Fig. 1.-Facular contrast versus viewing angle. This figure is similar to fig. 1 of Chapman (1970). The open and filled circles represent observations, from different sources, of facular contrast $\Delta I / I$ versus the cosine of the viewing angle $\theta$. The smooth curve is computed from the simplified facular model used in this paper. The diagram at the left shows the assumed geometry of a single facula. 
facular model, from which the smooth curve was computed, satisfies the following conditions: The excess radiation is emitted from a source region $750 \mathrm{~km}$ in diameter which extends upward from the level at which the facular optical depth is $\tau_{0}$. Inside the source region the source function is greater than that of the surroundings by an amount $\Delta B$. Optical depth within the source region decreases exponentially with respect to height with a scale height of $100 \mathrm{~km}$. The entire source region is above the top of the photosphere, so all emergent rays pass unattenuated through the adjacent solar atmosphere. The curve shown in the figure satisfies the condition

$$
\tau_{0} \Delta B=(0.03) I_{0}
$$

where $I_{0}$ is the photospheric intensity at the center of the solar disk.

As mentioned above, we require that the source region be optically thin for all emergent rays. The greatest optical thickness is for a horizontal ray, and since the diameter of the source region is 7.5 times the scale height, we require that

$$
(7.5) \tau_{0} \ll 1
$$

In order that the model be dynamically self-consistent, we require that the gas pressure $P$ at the base of the source region be equal to or less than the pressure at the top of the photosphere. Conditions at the top of the photosphere are characterized by $P \approx 10^{4}$ dyn $\mathrm{cm}^{-2}, T \approx 4600^{\circ} \mathrm{K}$, and $\tau \approx 0.004$ (Gingerich and de Jager 1968). Conditions at the base of the facular source region are then obtained from equation (1)

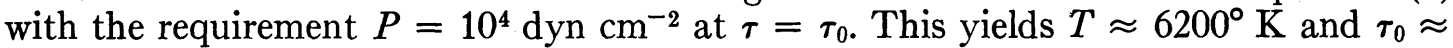
0.04 , which is incidentally consistent with equation (2). Note that optical depth within the facula is $\sim 10$ times greater than that of the surroundings. This is due to the fact that opacity increases sharply as the temperature increases. This model is similar in many respects to a more detailed model proposed by Chapman (1970), but is different from that of Rogerson (1961). Differences are due mainly to the fact that Rogerson did not take into account the temperature dependence of opacity; and did not consider the requirement of dynamic equilibrium. Gradients of the magnetic pressure undoubtedly influence the conditions for dynamic equilibrium, but the effect of the magnetic field is uncertain.

This model has the advantage of being a conceptually simple model which is consistent with recent high-resolution observations of faculae (fig. 1), and which yields a $\delta$ dependence of facular signal consistent with the $\delta$-dependence of Dicke and Goldenberg's points (fig. 2 of Ingersoll and Spiegel). However, because the $\delta$-dependence of Dicke and Goldenberg's points is not well determined, a certain freedom in the model is permitted. As presently formulated, the model predicts that a faint bright arc will be observed within $\sim 1^{\prime \prime}$ from the solar limb whenever the coverage of faculae exceeds 10 percent in the region on the limb. ${ }^{2}$ Such an arc is apparently not observed, which suggests that our model is not correct at the extreme limb $(\mu \lesssim 0.03)$; however, it is possible to formulate a slightly more realistic facular model which does not lead to bright arcs, but which still satisfies the $\delta$-dependence of Dicke and Goldenberg's points. Thus, if future observations should indicate that our facular model is in error at the extreme limb $(\mu \lesssim 0.03)$, faculae should still have the necessary $\delta$-dependence to contribute to Dicke and Goldenberg's oblateness measurement. To go further, one needs photometric observations of faculae at the extreme solar limb, and such observations have not been made.

\section{MEAN SIGNAL AMPLITUDE}

Our second major task is to estimate the average oblateness signal expected during 1966 as a result of faculae on the limb. Here the basic observable is $A$, the fractional area on the solar surface occupied by faculae. If we consider a unit area on the solar

2 This prediction of the model was noted by Dicke (1971b). 
surface, $A$ is the area occupied by faculae. Since the source region of each facula is optically thin, the excess power radiated per unit solid angle is independent of viewing angle, and is simply $A \tau_{0} \Delta B$. If we now consider a unit length along the circumference of the solar disk, then the area of the solar surface between the limb $(\mu=0)$ and the occulting disk $\left(\mu=\mu_{0}\right)$ is approximately $a \mu_{0}$, where $a$ is the mean solar radius. This means that the contribution to the oblateness signal (excess power per unit solid angle per unit circumferential length) due to faculae in the field of view is $A \tau_{0} \Delta B a \mu_{0}$. In the same units, the contribution to the oblateness signal measured by Dicke and Goldenberg is $I_{p}(r-a)$, where $I_{p}$ is the intensity of the photosphere at the edge of the occulting disk and $(r-a)$ is the inferred difference between the solar radius at the point of observation and the mean solar radius.

Dicke and Goldenberg measured the second Fourier harmonic of the light flux curve as they scanned around the circumference of the disk. Thus, the two quantities we wish to compare are

and

$$
\tau_{0} \Delta B \mu_{0} a^{2} \int_{0}^{2 \pi} A \cos 2 \phi d \phi
$$

$$
I_{p} a^{2} \int_{0}^{2 \pi}\left(\frac{r-a}{a}\right) \cos 2 \phi d \phi
$$

where $\phi$ is the angle from the pole measured counterclockwise around the solar disk. Our aim in this section is to derive numerical estimates of the above two expressions.

We first obtain estimates of the quantities in equation (3a). We define $\bar{A}$ as the average value of $A$ for the solar surface. Thus we have

$$
\bar{A}=\frac{1}{2} \int_{0}^{\pi} A(\phi) \sin \phi d \phi .
$$

It is observed that during 1966 most faculae occurred in the northern hemisphere in a narrow latitude band centered at latitude $90^{\circ}-\phi=23^{\circ}$. Accordingly, we take

$$
A(\phi)=\frac{2 \bar{A}}{\sin 67^{\circ}}\left[\delta_{\mathrm{D}}\left(\phi-67^{\circ}\right)+\delta_{\mathrm{D}}\left(\phi+67^{\circ}\right)\right],
$$

where $\delta_{\mathrm{D}}$ is the Dirac delta-function. Use of other more realistic distribution functions than the Dirac delta function has a negligible effect on the results. The value of $\bar{A}$ is obtained from Allen (1963). He gives $\bar{A}=38 \times 10^{-6} R_{\mathbf{Z}}$ and $\bar{A}=25 \times 10^{-6} R_{\mathrm{Z}}$ at sunspot minimum and maximum, respectively, where $R_{\mathbf{Z}}$ is the Zurich sunspot number. Interpolating halfway between sunspot minimum and maximum for 1966, we obtain

$$
\bar{A}=1.7 \times 10^{-3} \text {, }
$$

where we have used $R_{Z}=55$ (Solar Geophysical Data 1971).

The value of $\tau_{0} \Delta B$ is obtained from our idealized facular model, equation (1). Using this estimate of facular brightness together with Allen's estimate of $\bar{A}$ is perhaps the major source of uncertainty in this analysis. However, we feel that the resulting estimate of facular signal is a conservative one. For example, in estimating facular brightness, we have used Rogerson's data uncorrected for instrumental smearing, so the quoted contrast should more nearly represent the facula as a whole than its brightest parts. Our own estimates of $\bar{A}$ for 1966 , based on methods described in the next section, is approximately 3 times Allen's estimate. Although our estimate is probably less reliable than Allen's, it leads us to believe that the use of Allen's $\bar{A}$ with equation (1) gives a conservative estimate of facular signal. 
We now obtain an estimate of the mean value of Dicke and Goldenberg's oblateness signal, expression (3b). We assume

$$
\left(\frac{r-a}{a}\right)=-\left(\frac{\Delta r}{a}\right) \frac{1}{2} \cos 2 \phi,
$$

where $\Delta r$ is the inferred difference between the equatorial and polar radii of the Sun. The values of $\Delta r / a$ for each of the three values of $\delta$ are obtained from figure 3 of Dicke $(1970 b)$. The corresponding three values of $I_{p} / I_{0}$ are obtained from table 1 of Dicke $(1970 a)$ and from Dicke (1970b). All of these data can be adequately represented by the relation

$$
\frac{1}{\mu_{0}} \frac{I_{p}}{I_{0}} \frac{\Delta r}{a}=(1.0 \pm 0.3) \times 10^{-4},
$$

which is the equation of the $\delta^{1 / 2}$ curve in figure 2 of Ingersoll and Spiegel. Dicke (1970c, $1971 a$ ) has pointed out that $\delta$ as defined in this paper was not measured in the experiments, and that the data reductions that could yield $I_{p} / I_{0}$ have not been made. For $\delta$ we have used distances to the "extrapolated limb" (fig. 3 of Dicke 1970b), defined by a linear extrapolation of the light flux to zero. For $I_{p} / I_{0}$ we have used Dicke's estimates (table 1 of Dicke 1970a) based on Allen's (1963) limb-darkening curve. We are forced to use these values because more appropriate values are not available. Moreover, we feel that the error estimate of equation (8) covers the range of possible uncertainty in both $\delta$ and $I_{p} / I_{0}$, and that equation (8) is a sufficiently accurate representation of Dicke and Goldenberg's data.

We now compare the magnitude of the mean facular signal with that of the mean oblateness signal by combining the above equations and taking the ratio of the two expressions in equation (3). We obtain

$$
\text { Facular signal/Oblateness signal }=1.0 \text {. }
$$

The uncertainty in the above quantity is large, and the close agreement is fortuitous. Nevertheless, this estimate was made without the use of free parameters, and the close agreement indicates that faculae are potentially a major source of oblateness signal.

We close this section with a short discussion of the effect of sunspots, using an approach similar to that above. Allen (1963) gives estimates of $\bar{A}$ and $\Delta I / I$ for sunspot umbrae and penumbrae as functions of $R_{\mathrm{Z}}$. According to Allen, the projected area of a sunspot is proportional to $\mu$, and the effective contrast is independent of $\mu$. With these assumptions, the ratio of mean sunspot signal to mean facular signal is proportional to $\mu_{0}$; the mean value of this ratio for all of Dicke and Goldenberg's data is - 0.14 . However, there is some evidence (e.g., Wilson and McIntosh 1969), that the umbra disappears as the sunspot approaches the limb, in which case the mean ratio of sunspot signal to facular signal may be only -0.08 . We conclude that sunspots have only a small effect on the mean oblateness signal.

\section{SIGNAL FLUCTUATIONS}

We now examine the time dependence of the facular oblateness signal, and show that fluctuations from the mean curve are correlated with observed fluctuations. In this study we have used photographs of the Sun, obtained daily at the San Fernando Observatory during the summer of 1966, to determine the fractional area $A$ occupied by faculae on the limb. Absolute values of $A$ obtained in this way are uncertain to an unknown scaling factor, so these data have been used only to establish correlation. With a more refined approach, it should be possible to determine the facular oblateness signal photometrically from photographs alone. 
The fractional area $A$ and mean latitude occupied by faculae were estimated by eye using a Stonyhurst grid. Values of $A$ were determined for standard zones extending $10^{\circ}$ in latitude by $15^{\circ}$ in longitude, in a longitude band $20^{\circ}-35^{\circ}$ from the limbs. This longitude band was chosen for best visibility of faculae and minimum foreshortening. Its width was chosen to cover 1 day of solar rotation without danger of undersampling. The center of this band is approximately 2 days' rotation from the limb, so conditions at the limb were inferred by shifting the data 2 days.

The principal uncertainty is a systematic or personal error in determining the coverage of faculae. It is assumed that this error is linear, such that values of $A$ obtained in this way are proportional to the true values. A second source of error is the intrinsic change of faculae during the two days of rotation between the limb and the standard zone. A third source of error is our use of a constant-longitude zone, rather than a zone of constant $\mu$ as in the oblateness experiments. Other sources of error include: wide variation in picture quality, use of neighboring zones to fill in for missing days of the photo record, and different times of observation on certain days. These errors are potentially large; their net effect is to degrade the data, and thereby to reduce the size of the correlation. An "answer-oriented" personal bias seems very unlikely, simply because it was not possible to guess what effect any given set of measurements would have on the oblateness signal.

In Dicke and Goldenberg's experiment, only the diagonal component was used in computing oblateness. This is the component along the NW-SE direction, using the standard astronomical convention. If $p$ is the angle measured counterclockwise from geographic North to the Sun's rotation axis, the part of the diagonal component due to faculae is

$$
\left(\frac{\Delta r}{a}\right)_{d}=-f \int_{0}^{2 \pi} A \sin [2(p+\phi)] d \phi,
$$

where $f$ is an unknown scaling factor. To eliminate the dependence on $f$, we shall express the daily facular signals obtained from equation (10) in terms of the mean signal, obtained in the following paragraph.

We assume that the ensemble mean $\langle A\rangle$ is a steady function of $\phi$, symmetric about the solar rotation axis $\phi=0$. The mean diagonal component due to faculae is then

$$
\left\langle\left(\frac{\Delta r}{a}\right)_{d}\right\rangle=-f \sin 2 p \int_{0}^{2 \pi}\langle A\rangle \cos 2 \phi d \phi,
$$

which is a function of day of observation through the factor $\sin 2 p$. Thus we may choose $f$ such that the mean curve for all the data (1966 May 30-October 9) is exactly $\sin 2 p$, with constant of proportionality equal to one. Table 1 gives the daily facular signals obtained from equation (10) with the constant $f$ determined in this way. We define the daily residuals as differences between the data of table 1 and the mean curve, sin $2 p$. The root mean square (rms) residual, expressed in these units, is then a dimensionless measure of signal variability, which we shall call the noise/signal ratio.

Dicke and Goldenberg's points, with which we wish to compare the present data, represent $\sim 2$-week averages for nine observation periods during the summer of 1966 . We do not know the daily or hourly signals, or exactly how these signals were combined to form 2-week averages. Dicke (1971b) has given us the initial and final dates of each 2-week period, as well as the individual dates of observation for seven of the nine periods (see table 1). Data from the first and last observation periods are less reliable than those from the other periods, according to Dicke (1971b). However, if we exclude these data, our sample size is limited to seven points; and although the data may be more reliable, the test of significance may be less reliable for the smaller sample. Accordingly, we have processed the data in two different ways. Our first approach has been to form averages 
TABLE 1

Daily Oblateness Signal Based on Solar Faculae in 1966

\begin{tabular}{|c|c|c|c|c|c|}
\hline \multirow[b]{2}{*}{ DAY } & \multicolumn{5}{|c|}{ Oblateness Signal } \\
\hline & June & July & August & September & October \\
\hline $\begin{array}{r}-1 \ldots \\
0 \ldots \\
1 \ldots \\
2 \ldots \\
3 \ldots \\
4 \ldots \\
5 \ldots \\
6 \ldots \\
7 \ldots \\
8 \ldots \\
9 \ldots \\
10 \ldots \\
11 \ldots \\
12 \ldots \\
13 \ldots \\
14 \ldots \\
15 \ldots \\
16 \ldots \\
17 \ldots \\
18 \ldots \\
19 \ldots \\
20 \ldots \\
21 \ldots \\
22 \ldots \\
23 \ldots \\
24 \ldots \\
25 \ldots \\
26 \ldots \\
27 \ldots \\
28 \ldots \\
29 \ldots \\
30 \ldots \\
31 \ldots \\
\end{array}$ & $\begin{array}{l}-0.38^{\mathrm{a}} \dagger \\
-0.09 \\
-1.17 \\
-0.37 \\
-0.20 \\
+0.09 \\
-0.51 \\
-1.15 \\
+0.16 \\
-0.52 \\
+0.81 \\
-0.01 \\
-0.01^{\mathrm{b}} \\
+0.06^{\mathrm{a}} \\
-0.39 \\
-0.09 \\
-0.01^{*} \\
-0.46 \\
+0.08 \\
-0.89 \\
+0.37 \\
-0.08^{*} \\
-0.31^{*} \\
-0.60^{*} \\
-0.31^{*} \\
-2.39^{*} \\
-0.81^{*} \\
-0.33^{\mathrm{b}} \\
+0.13^{\mathrm{a}} * \\
+0.65 \\
+0.31 \\
-0.48^{*} \\
\ldots\end{array}$ & $\begin{array}{l}\ldots \\
-1.82^{*} \\
-0.73^{*} \\
-0.03^{*} \\
-0.30^{*} \\
+0.64 \\
+0.97 \\
+1.29 \\
+0.12^{*} \\
+2.48^{*} \\
+2.16^{2} \\
+0.37^{\mathrm{b}} \\
-0.82^{\mathrm{a}} * \\
+0.97^{*} \\
+1.62 \\
+1.32 \\
-0.83^{*} \\
-0.03^{*} \\
+0.51^{\mathrm{b} *} \\
-0.41^{\mathrm{a}} \\
-0.75^{*} \\
+0.94^{*} \\
+0.04^{*} \\
-1.19^{*} \\
-2.04^{*} \\
-0.46^{*} \\
+0.80^{*} \\
-0.13^{*} \\
-0.51^{\mathrm{b}} \\
-0.36^{\mathrm{a}} \\
-0.06 \\
-0.08^{*}\end{array}$ & $\begin{array}{l} \\
\ldots \\
+1.52^{*} \\
+1.42 \\
+0.25^{*} \\
+0.30 \\
+0.41 \\
+1.90^{*} \\
+2.42 \\
+1.64 \\
+1.76 \\
+1.19 \\
+0.42 \\
+0.37 \\
+0.17^{*} \\
+0.28 \\
+0.03 \\
+0.20^{\mathrm{b}} \\
-0.10^{\mathrm{a}} * \\
-0.39^{*} \\
-0.71^{*} \\
+0.12 \\
+0.53^{*} \\
+0.54 \\
+0.33 \\
+0.12^{*} \\
+0.17 \\
+0.79^{*} \\
+0.02^{*} \\
+0.31^{*} \\
+0.86^{\mathrm{b}} \\
+1.71 \\
+0.68^{\mathrm{a} *}\end{array}$ & $\begin{array}{l}\ldots \\
+0.50 \\
+1.34 \dagger \\
+1.87 \dagger \\
+2.40 \\
+1.41^{*} \\
+0.71 \dagger \\
+0.79^{*} \dagger \\
+0.14 \\
+1.06 \\
+0.21^{*} \\
+0.16^{*} \\
+0.69 \\
+0.74 \\
+0.28 \\
+0.08 \\
+0.10^{*} \\
+0.27 \\
+1.65^{*} \\
+1.11 \\
+0.51 \\
+0.21 \\
+0.65 \\
+0.41^{\mathrm{b} *} \\
+0.15^{\mathrm{a}} \\
+1.18 \\
+2.13 \dagger \\
+1.73 \\
+1.77 \\
+0.58 \\
+0.83 \dagger \\
+\ldots\end{array}$ & $\begin{array}{c}\cdots \\
+0.43 \dagger \\
+1.88 \\
+3.05 \\
+0.73 \\
+0.82 \dagger \\
+0.36 \\
+0.47 \\
+0.12 \\
+0.45^{b}\end{array}$ \\
\hline
\end{tabular}

Note.-The symbols ${ }^{a}$ and ${ }^{b}$ denote the beginning and end of each observation period. An asterisk denotes a day of observation, according to Dicke (1971b). A dagger denotes a missing half-day in the photo record; such days were given halfweight in the analysis. The data are scaled to $\sin 2 p$, and the rms daily residual (difference of the data from $\sin 2 p$ ) is approximately 0.81 . June -1 and 0 correspond to May 30 and 31 , respectively.

of the data in table 1 for each of the nine observation periods using all the days in each period. Our second approach has been to construct averages for seven of the nine periods using only those days given us by Dicke. Thus, from the data of table 1 we have constructed two sets of numbers, each of which is to be compared with the data obtained from figure 3 of Dicke (1970b).

Two steps are required in order to cast the data from figure 3 of Dicke (1970b) into a form suitable for comparison with facular data. First, it is necessary to correct for the oblateness associated with the surface rotation of the Sun. This accounts for about one-fifth of the observed signal. Accordingly, the values of $(\Delta r / a)_{d}$ were corrected by subtracting $10^{-5}$ sin $2 p$ from each data point. Second, it is necessary to average the data with respect to the three values of $\delta$. As mentioned earlier (cf. eq. [8]), the quantity $\left(1 / \mu_{0}\right)\left(I_{p} / I_{0}\right)(\Delta r / a)_{d}$ is approximately independent of $\delta$; moreover, it can be shown 
(e.g., by comparing the three error estimates in fig. 2 of Ingersoll and Spiegel) that the variance of this quantity is also approximately independent of $\delta$. Therefore, in averaging with respect to $\delta$ it is appropriate to weight each value of $(\Delta r / a)_{d}$ by the appropriate value of $\left(1 / \mu_{0}\right)\left(I_{p} / I_{0}\right)$. In this way, a single set of nine numbers was constructed from the data in figure 3 of Dicke (1970b). Henceforth, we shall refer to this set of numbers as the "oblateness" data, and the numbers constructed by averaging the data in table 1 as the "facular" data.

In figure 2 the oblateness data are compared with the facular data computed from table 1 using all the days in each observation period. The two sets of data are both scaled so that the best-fitting curve is exactly $\sin 2 p$, with constant of proportionality equal to unity. In computing the best-fitting curve, we minimize the sum of squares of weighted residuals, with weights assigned as follows: For the nine facular points, each weight is proportional to the square root of the total number of days in the interval. For seven of the nine oblateness points, each weight is proportional to the square root of the number of observation days in the interval, as communicated by Dicke (1971b). For the remaining two oblateness points, each weight is equal to the average weight of the other oblateness points. If the errors from different days are uncorrelated, this weighting ensures that all days are treated equally in the analysis based on 2-week averages. Similarly, in constructing the two sets of nine residuals the values of $\sin 2 p$ were averaged over all days in each interval for facular points, and over the observation days in each interval for oblateness points. This ensures that both sides of equations (10) and (11) are treated in the same way in constructing 2-week averages.

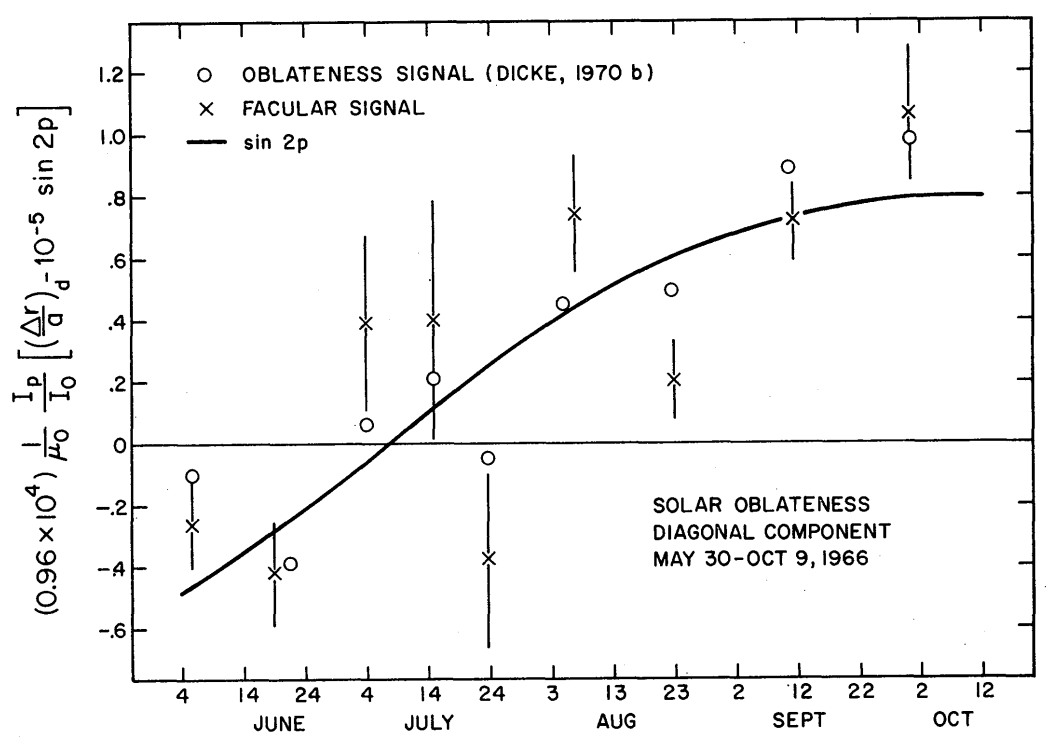

FIG. 2.-Comparison of the observed oblateness signal with the facular oblateness signal. The circles were computed as described in the text from Dicke and Goldenberg's data (fig. 3 of Dicke 1970b) with the part due to surface rotation removed. The crosses represent the means for nine observation periods of all the facular data given in table 1 . The solid curve represents the temporal variation of sin $2 p$, where $p$ is the angle of the solar rotation axis from geographic north. Both the circles and the crosses have been scaled so that the best-fitting curve to each data set is exactly $\sin 2 p$. Each set of vertical bars is computed as an error of the mean using the rms variation of the daily facular signal from the mean facular signal for that observation period. The abscissae of the facular points differ slightly from those of Dicke and Goldenberg's points because the former represent averages over all days and the latter represent averages over a limited number of days in each observation period. The correlation coefficient of the residuals (the differences between the plotted points and $\sin 2 p$ ) for the two sets of data is 0.75 , which is statistically significant at the 1 percent level. The rms residual of the facular points is 1.63 times the rms residual of Dicke and Goldenberg's points. 
The results of the comparison of the data shown in figure 2 can be summarized as follows: The correlation coefficient of the residuals weighted as described above is 0.75 , and the value of the rms residual (weighted mean) for the facular data is 1.63 times the corresponding value for the oblateness data. This correlation coefficient is significant at the 1 percent level, by which we mean that positive values of the correlation coefficient greater than this value will occur less than 1 percent of the time for uncorrelated data. We conclude that the correlation coefficient is statistically significant, which suggests that faculae are the major source of noise in Dicke and Goldenberg's observations. Statistical significance and ratio of residuals $=1.0$ would imply that faculae were also the major source of signal in the oblateness observations. The fact that the ratio of residuals is 1.63 suggests that faculae contribute only $\sim 60$ percent of the oblateness signal. However, other interpretations are possible. These will be discussed in the next section.

We have also compared the oblateness data with the facular data computed from table 1 using only the days of observation given us by Dicke. In this case, both the facular residuals and the oblateness residuals were weighted according to the number of observation days in the interval. The comparison involves only seven of the nine observation periods, as explained previously. For these data, the correlation coefficient is 0.80 , and the ratio of residuals is 3.34 . This value of the correlation coefficient is somewhat greater than the previous value, but because of the smaller number of data points the results are significant only at the 2 percent level. This value of the ratio of residuals suggests that faculae contribute only $\sim 30$ percent of the oblateness signal.

It is difficult to include the effect of sunspots in the above analysis, mainly because the unknown scaling factor $f$ (eq. [10]) may be different for sunspots and faculae. One approach is to require that the mean signal due to sunspots be a certain fraction of the mean signal due to faculae. When this fraction is taken to be -0.10 , which is reasonable according to the discussion in the preceding section, the correlation coefficient and ratio of residuals both decrease by less than $\sim 5$ percent. Thus the mean effect of sunspots is small, although on several days the sunspot signal was nearly equal and opposite to the facular signal. The effects of sunspots were not included in table 1 or in the discussion of correlation coefficients.

We close this section with a discussion of errors. Each number in table 1 is the result of averaging two separate readings of the photographs. When these two readings are compared, we obtain an uncertainty estimate for the numbers of table 1 which is based on the lack of repeatability of our measurements. We find, first, that the correlation coefficient of the daily residuals from $\sin 2 p$ between one reading and the other is 0.83 . Second, we find that the rms difference between the daily facular signal from one reading and that from the average of the two readings is 0.25 in the units of table 1 . This is to be compared with the rms daily residual (difference from $\sin 2 p$ ) of the data in table 1 , which is 0.81 . We also find that larger signals tend to have errors larger than the mean, the error becoming, on the average, $\pm \frac{1}{4}$ of the signal for larger signals. Thus, although there is an appreciable error due to lack of repeatability, we conclude that much of the variability of the facular signal is due to intrinsic solar variations. We suggest that some of the variability of the oblateness signal is also due to solar variations.

Similarly, we may also find the uncertainty, due to lack of repeatability, in each of the nine facular points of figure 2 . For each interval, this uncertainty is approximately $(N-1)^{-1 / 2}$ times the rms difference between one reading of the daily facular signal and the mean of both readings, where $N$ is the number of days in the interval. These uncertainties range from \pm 0.04 to \pm 0.15 , the (rms) average being about \pm 0.07 . For comparison, the (rms) average of the length of the error bars in figure 2 is about \pm 0.23 . Each of these error bars is $(N-1)^{-1 / 2}$ times the rms difference between the daily facular signal of table 1 and the mean signal for the interval. Again we conclude that most of the signal variability is due to intrinsic solar variation. 
Although we cannot determine the magnitude of possible systematic errors, we assume that they are at least as large as the errors due to lack of repeatability. Thus the numbers of table 1 may be uncertain by a factor of 2 for comparison with the daily oblateness signal. We shall discuss possible systematic errors more fully in the next section.

\section{v. DISCUSSION}

We believe ours is the first published criticism of Dicke and Goldenberg's oblateness determination which is based entirely on empirical data. Although we find that faculae probably do account for at least part of the excess oblateness signal (and may account for all of it), there are several significant uncertainties in the comparison we have made. In $\S$ III we made a conservative estimate of the mean facular signal and found that it was comparable to the mean oblateness signal. However, there was a significant uncertainty in the estimate of the mean area $\bar{A}$ of faculae on the solar surface. In $\S$ IV we found that fluctuations in the facular signal were significantly correlated with fluctuations in the oblateness signal. However, the ratio of noise/signal for the facular data was about 1.63 times that for the oblateness data. Moreover, when only the "correct" days of observation were used, the value of this ratio increased to 3.34. This increase is due largely to the intrinsic variability of the facular signal: use of fewer days from which to construct 2-week averages leads to an increase in the noise/signal ratio. Nevertheless, it appears that the facular signal is considerably more variable than the oblateness signal. We now speculate on possible causes of this apparent discrepancy.

First, it is possible that the faculae we have measured account for only $30-60$ percent of the oblateness signal but still account for most of the noise. The remainder of the signal would have to have a low noise/signal ratio in order to explain the results of $\S \mathrm{IV}$. True gravitational oblateness, somewhat smaller than that inferred by Dicke and Goldenberg, could explain this part of the signal; but a background of weak faculae, undetected by us, could also explain these results. It is also possible that our errors of measurement are larger than our estimated errors given in the preceding section, or that our measurements are very nonlinear and give too much weight to large facular areas relative to small ones. Either of these effects might explain the excess noise/ signal in the facular data. Finally, some of the discrepancy may arise because we may not have followed exactly the same procedures as Dicke and Goldenberg followed in constructing their 2-week averages. It is difficult for us to assess the importance of these possible differences, and therefore we feel it is fruitless to refine our analysis further until more of the oblateness data are available.

When the daily oblateness data become available, it will be useful to compare them with table 1 . The large increase in the number of degrees of freedom should make possible a more refined comparison of the two signals. However, one should bear in mind certain reservations about the data in table 1 . First, the measurement error in our visual estimates of facular signal is high; our eye estimates certainly could not have the accuracy of a photoelectric device. Second, our measurements refer to a longitude band $15^{\circ}$ wide, whereas the oblateness observations refer to bands of $\mu=\mu_{0}$, corresponding to $7^{\circ}, 9^{\circ}$, and $12^{\circ}$ of longitude at the equator. Thus, our longitude band is generally wider (except near the poles) than the observation bands used in the oblateness experiment. In comparing the data of table 1 with Dicke and Goldenberg's measurements, one should assume that the numbers of table 1 are uncertain by a factor of about 2, and the day to which each number is assigned is uncertain by about \pm 0.5 day. We intend to improve our facular model and our estimates of daily facular signal with future observations and analysis.

Dicke (1970b) mentions observations from 1967 which gave the same oblateness with comparable precision as Dicke and Goldenberg's observations of 1966. However, we would expect a greater oblateness signal in 1967 due to increased solar activity. The method outlined in $\S$ III of this paper predicts a 57 percent increase in the facular part 
of the signal, which yields an expected oblateness $(\Delta r / a) \sim 7.3 \times 10^{-5}$ for 1967 , if one assumes that the entire excess signal is caused by faculae. It would be interesting to compare this estimate with Dicke and Goldenberg's 1967 observations when these become public.

We suggest that future observations of solar oblateness be made at widely different parts of the 11-year solar cycle, especially at the time of solar minimum. These observations should also be made at wavelengths referring to different levels in the solar atmosphere, and at observing sites where a wide range of $\delta$ 's is possible. High-resolution photographs of the Sun should be obtained concurrent with oblateness measurements to determine the presence of solar faculae in the zone being measured. Finally, in future experiments of the Dicke and Goldenberg type, provision should be made for storing harmonics of the signal higher than the second so that the Fourier signature of faculae can be explicitly detected. In short, every effort must be made to separate the effects of brightness variation from those of true gravitational oblateness.

We gratefully acknowledge support of this research by company funds of the Aerospace Corporation and by the National Aeronautics and Space Administration under grant NGL 05-002-003. We have also benefited from suggestions and criticisms of this paper by Professor Dicke.

\section{REFERENCES}

Allen, C. W. 1963, Astrophysical Quantities (2d ed.; London: Athlone Press).

Chapman, G. A. 1970, Solar Phys., 14, 315.

Dicke, R. H. $1970 a, A p . J ., 159,1$.

. 1970b, Ann. Rev. Astr. and A p. 8, 297.

- 1970c, private communication.

. 1971a, private communication.

1972, A p. J., 175, 831.

Dicke, R. H., and Goldenberg, H. M. 1967, Phys. Rev. Letters, 18, 313.

Gingerich, O., and de Jager, C. 1968, Solar Phys., 3, 5.

Ingersoll, A. P., and Spiegel, E. A. 1971, Ap.J. 163, 375.

Rogerson, J. B. 1961, Ap. J., 134, 331.

Solar Geophysical Data. 1971 (U.S. Department of Commerce, National Oceanic and Atmospheric Administration compiled by the Aeronomy and Space Data Center, Boulder, Colorado).

Wilson, P. R., and McIntosh, P. S. 1969, Solar Phys., 10, 370.

Note added in proof.-We are preparing a detailed analysis of the daily signals, using the data contained in Dr. Dicke's paper, "Faculae and the Solar Oblateness" (Dicke 1972). 
